\title{
Carbon dioxide absorption heat in liquid-liquid and solid-liquid phase-change solvents using continuous calorimetry
}

Khuyen V. B. Tran ${ }^{1}$, Ryuya Ando ${ }^{2}$, Tsuyoshi Yamaguchi ${ }^{3}$, Hiroshi Machida ${ }^{1 *}$, Koyo

\section{Norinaga $^{1}$}

${ }^{1}$ Department of Chemical Systems Engineering, Nagoya University, Japan

${ }^{2}$ Department of Molecular Design and Engineering, Nagoya University, Japan

${ }^{3}$ Department of Materials Process Engineering, Nagoya University, Japan

S1. Table S1. Absorption heat of amine solvents in comparison with literature data. 


\begin{tabular}{|c|c|c|c|}
\hline Solutions & Ref. & 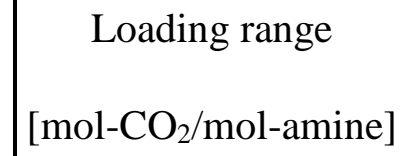 & $\begin{array}{c}\text { Absorption heat } \\
{\left[\mathrm{kJ} / \mathrm{mol}^{-} \mathrm{CO}_{2}\right]}\end{array}$ \\
\hline \multirow{7}{*}{ MEA } & This study & $0-0.500$ & 82.68 \\
\hline & 10 & $0.451-0.494$ & 84.07 \\
\hline & 10 & $0.463-0.507$ & 73.57 \\
\hline & 9 & $0.406-0.497$ & 77.36 \\
\hline & 14 & $0.4-0.6$ & 86.90 \\
\hline & $*$ & $0-0.565^{b}$ & 88.91 \\
\hline & 13 & $0-0.532$ & 88.00 \\
\hline \multirow{3}{*}{ EAE30 } & This study & $0-0.545$ & 78.04 \\
\hline & 4 & $0-0.710^{\mathrm{a}}$ & 69.00 \\
\hline & 13 & $0-0.682$ & 78.00 \\
\hline \multirow{3}{*}{ AMP25 } & This study & $0-0.651$ & 76.99 \\
\hline & 12 & $0.548-0.652$ & 68.94 \\
\hline & 11 & $0.594-0.655$ & 69.12 \\
\hline EAE80 & \multirow[t]{4}{*}{ This study } & $0-0.397$ & 82.10 \\
\hline EAE DEGDEE water 306010 & & $0-0.474$ & 84.98 \\
\hline EAE DEGDEE 3070 & & $0-0.381$ & 98.99 \\
\hline AMP50 & & 0.197 & 87.39 \\
\hline
\end{tabular}


Explanation: ${ }^{\mathrm{a}}: \mathrm{p}_{\mathrm{CO} 2}=15 \mathrm{kPa} ;{ }^{\mathrm{b}}:$ at $25^{\circ} \mathrm{C}$.

* Kim, Y. E.; Lim, J. A.; Jeong, S. K.; Yoon, Y. Il; Bae, S. T.; Nam, S. C. Comparison of Carbon Dioxide Absorption in Aqueous MEA, DEA, TEA, and AMP Solutions. Bull. Korean Chem. Soc. 2013, 34 (3), 783-787. https://doi.org/10.5012/bkcs.2013.34.3.783. 